\title{
Colorimetric Ethanol Indicator Based on Instantaneous, Localized Wetting of a Photonic Crystal
}

\section{Citation}

Yu, Yanhao, Soeren Brandt, Natalie J. Nicolas, and Janna Aizenberg. "Colorimetric Ethanol Indicator Based on Instantaneous, Localized Wetting of a Photonic Crystal." ACS Applied Material and Interfaces 12, no. 1 (Jan 2020): 1924-1929. 10.1021/acsami.9b19836

\section{Permanent link}

http://nrs.harvard.edu/urn-3:HUL.InstRepos:42165869

\section{Terms of Use}

This article was downloaded from Harvard University's DASH repository, and is made available under the terms and conditions applicable to Open Access Policy Articles, as set forth at http:// nrs.harvard.edu/urn-3:HUL.InstRepos:dash.current.terms-of-use\#OAP

\section{Share Your Story}

The Harvard community has made this article openly available. Please share how this access benefits you. Submit a story. 


\title{
A Colorimetric Ethanol Indicator based on Instantaneous, Localized Wetting of a Photonic Crystal
}

\author{
Yanhao $\mathrm{Yu}^{1,2}$, Soeren Brandt ${ }^{1,2}$, Natalie J. Nicolas ${ }^{1,2}$, Joanna Aizenberg ${ }^{1-4}$
}

1. John A. Paulson School of Engineering and Applied Sciences, Harvard University, Cambridge, MA, USA, 02138. 2. Wyss Institute for Biologically Inspired Engineering, Harvard University, Cambridge, MA, USA, 02115. 3. Department of Chemistry and Chemical Biology, Harvard University, Cambridge, MA, USA, 02138. 4. Kavli Institute for Bionano Science and Technology, Harvard University, Cambridge, MA, USA, 02138.

\begin{abstract}
Easy-to-use sensors for ethanol solutions have broad applications ranging from monitoring alcohol quality to combating underage drinking. Although there are a number of electronic and colorimetric sensors available for determining alcohol concentration, there is currently no device that can concurrently provide a prompt, well-defined, quickly recoverable readout and remain readily affordable. Here, we developed a field-ready, colorimetric indicator that provides fast, clear identification of ethanol/water mixtures between 0 to $40 \%$ based on the discoloration of a wetted photonic crystal. We cooperatively exploit the iridescence and the geometrical gating in silica inverse opal films (IOF), together with a finetuned surface chemistry gradient, to distinguish ethanol concentrations by their wettability patterns in different segments of the IOF. The resultant all-in-one colorimetric sensor delivers a striking and instantaneous optical response at ethanol concentration as low as $5 \%$. We further improve the ease of use by seamlessly integrating this colorimetric platform with drinking glassware (a glass stirrer and a vial). This research provides an optimal means for colorimetric ethanol detection and is a step towards the immersible sensing of diverse molecules (e.g. biomarkers) in aqueous solutions without expensive laboratory tests.
\end{abstract}

Key words: ethanol detection, colorimetric, structural coloration, wetting, W-Ink 


\section{Introduction}

Measuring ethanol content in a fast and straightforward manner has profound implications for quality control and legitimate consumption of alcoholic beverages. A longstanding motivation for ethanol differentiation originates from preventing adulteration and protecting the consumer from alcohol with inaccurately advertised alcohol content. ${ }^{1,2}$ Another growing area of interest lies in sensing and preventing underage drinking by adding liquor to non-alcoholic beverages. ${ }^{3,4}$ Both of these scenarios demand easy, immediate, on-site sensing of ethanol, which precludes using such traditional in-laboratory techniques as gas chromatography, liquid chromatography, mass spectrometry and UV-vis spectrometry. ${ }^{5-11}$ Handheld electronic and optoelectronic noses are prevalent in vapor ethanol detection but are less compatible with liquid phase and have relatively high costs. ${ }^{12-14}$ Colorimetric sensing is inexpensive and easy to use and thus has become a popular direction for developing on-site ethanol indicators. A large portion of colorimetric sensors operate based on building blocks that have adaptive optical absorption or fluorescence in response to ethanol or other molecules in liquors (e.g., aldehydes and ketones). ${ }^{15-17}$ One representative process is the release of fluorescent dyes that are pre-encapsulated in an ethanol/waterresponsive polymer matrix. ${ }^{18,19}$ This type of color change is generally irreversible and takes minutes to reach steady state, which is inconvenient for the user.

In addition to molecular dyes, photonic crystals have also been explored for ethanol detection. These crystals get their coloration from periodic nanostructures having refractive indices different from that of the surrounding medium. These structures lead to bright, iridescent colors that depend on the size and shape of the structure as well as the wavelength and direction of the incoming light. ${ }^{20}$ Polymeric photonic crystals take advantage of geometric deformation (swelling or de-swelling) in ethanol-water mixtures and discern ethanol concentrations by the shift of peaks in the UV-Vis spectrum. ${ }^{21}$ However, this size-induced color change is challenging to judge by eye due to the subtle variation in color and the angle-dependence of the color. ${ }^{22}$ By combining structural coloration with selective wettability, our group introduced the Wetting-in-color (W-Ink) platform that conforms well to the needs of quick field-testing of ethanol. ${ }^{23,24}$ 
W-Ink uses interconnected highly ordered inverse opal films (IOFs) and silane surface functionalization to control the infiltration of liquid into the structure. This infiltration leads to the disappearance of color when the refractive index contrast between the IOF matrix and the liquid is small. The IOF is infiltrated when its surface is sufficiently lyophilic - that is, when its surface chemistry is compatible with the infiltrating liquid making wetting and infiltration energetically favorable. With proper design of the hydrophobicity we can use W-Ink to determine the ethanol concentration of a solution based on the appearance and disappearance, rather than the subtle change, of color. To date, W-Ink-like sensors have been used to effectively discriminate between common organic solvents and high ethanol concentration solutions. ${ }^{23-37}$ However, chemical functionalization with fluorinated and highly hydrophobic alkyl silanes used in these sensors fails at discriminating higher-surface-tension liquids, such as low-concentration solutions of ethanol, and requires the development of an alternative, more hydrophilic surface chemistry capable of displaying concentration-specific wettability at low concentrations of ethanol. In this paper, we report a reusable, field-ready W-Ink colorimetric indicator for detecting low- to mid-range concentrations of ethanol in aqueous solution, typical for alcoholic beverages. The color change is quick, clear, and easy to recover. We have developed surface functionalizations using silane chemistry to detect a series of ethanol/water mixtures with ethanol concentrations ranging from 0 to $40 \%$. We then used a facile dipcoating process to sequentially coat these silanes onto neighboring sections of the IOF to vary the hydrophobicity along the length of the sensor. Each segment has a wetting threshold corresponding to a specific concentration of ethanol and thereby provides a convenient readout of the ethanol concentration by the presence or absence of the color. The IOF film was later patterned on glass rods and vials, to demonstrate field-ready practical ethanol indicators.

\section{Results and Discussion}

Both natural and synthetic direct opals are formed when colloidal particles assemble into a threedimensional, close-packed array. To make an inverse opal, a direct opal is used as a sacrificial template that is first infiltrated with a matrix material and then removed. In this study, we fabricated thin film 
inverse opals using evaporation-induced co-assembly of a polystyrene colloidal template and silica matrix material formed from the polycondensation of tetraethyl orthosilicate (TEOS). ${ }^{38}$ After co-assembly, the colloids are removed by calcination at $500{ }^{\circ} \mathrm{C}$. This process yields highly ordered and crack-free IOF (Figure 1a). The IOF typically consists of five and a half layers of pores and has a film thickness of $\sim 1.5$ $\mu \mathrm{m}$ (Figure 1b). The pores of the IOF form a highly interconnected network with small 'gating' necks, roughly one third the size of the pore (Figure 1c). Through these necks, a wetting liquid will infiltrate the pores of the IOF, which is the basis of the detection mechanism employed here. The resolution of the detection is ultimately limited by the uniformity of the neck angles. The colloidal co-assembly process employed to create the IOF results in a very narrow distribution of pore neck angles of less than three degrees. $^{23,24}$

IOF photonic crystals with pores on the order of $200-600 \mathrm{~nm}$ display strong iridescent color under ambient conditions. A typical silica sample prepared from $360 \mathrm{~nm}$ templating colloids has a greenish blue color when observed at normal incidence in diffuse lighting (Figure 1d(i)). This color is produced by the angle- and wavelength-dependent reflection of visible light at the top surface and inside of the IOF. The intensity of the color is related to the reflectance of the material (i.e., 5.5 layers of inverse opal) in air $(51.5 \%)$ and when infiltrated by water $(3.9 \%)$, which we calculated using a one-dimensional transfer matrix approach. ${ }^{39}$ Therefore, the color is easily extinguished by infiltrating the IOF with water (Figure 1d(ii)). Owing to the natural hydrophilicity of the pristine silica surface, water readily wets the surface and fills the pores of the IOF. By tuning the inherent hydrophilicity of the silica surface, we can control the extent to which the IOF is wetted and consequently how much of the IOF is rendered colorless. Since the refractive index of ethanol $\left(n_{\text {ethanol }} \approx 1.36\right)$ is close to that of water, any ethanol-water mixtures that penetrate into the IOF will reduce the reflectance to a similar degree. Once the mixture evaporates from the IOF, the colorful appearance is restored as air refills the pores. This can be repeated many times with no degradation as long as the structure of the IOF remains intact. 
To build a functional sensor using the strong and reversible color contrast of IOF between the dry and wetted states, we have created an IOF with step-wise increasing surface hydrophobicity (Figure 1e). This sensor exhibits bright iridescence in air, but when placed into an ethanol-water mixture, the sections where surface chemistry favors wetting are infiltrated and lose color while the remaining sections stay non-wetted and colorful. Each ethanol/water mixture is expected to have a unique benchmark on the IOF. These benchmarks establish a scoring system that indicates a series of ethanol percentages.

A non-wetted region exists because the necks between pores inside the IOF prevent the propagation of a liquid with a contact angle above a critical value. This gating mechanism is based on capillary action pushing the analyte liquid into the IOF pores. This force is the result of the Laplace pressure across the interface between the liquid and the air filling the dry pore. In general, an interface with positive Gaussian curvature has a positive Laplace pressure and the liquid infiltrates the pore. In a straight capillary, the curvature of the interface depends on the radius of the capillary and the contact angle of the liquid. Here, as the liquid front propagates through the pore, the apparent radius changes, as does the angle of the surface. As a result, the liquid's ability to infiltrate depends on the relationship between the intrinsic contact angle $\theta_{c}$ of the liquid and the opening angle $\varphi_{0}$ of the neck between two pores (Figure $2 \mathrm{a}$ ). ${ }^{23}$ When the contact angle is larger than the opening angle $\left(\theta_{c} \geq \varphi_{0}\right)$, the interface has zero Gaussian curvature at the neck, thus preventing further infiltration and leaving the following pores dry and colorful (Figure 2a(i)). When the contact angle is smaller $\left(\theta_{c}<\varphi_{0}\right)$, the liquid meniscus remains curved and continues to move through the neck, filling the pores and leading to discoloration (Figure 2a(ii)).

For the IOF shown in Figure 1c, the neck opening angle $\varphi_{0}$ was calculated to be $\sim 21^{\circ}$. In order to distinguish two ethanol concentrations, one of the contact angles $\left(\theta_{c}\right)$ of the mixtures needs to be larger and the other smaller than the opening angle $\varphi_{0}$. The surface tensions of ethanol-water mixtures decrease monotonically with increasing ethanol concentration, especially at ethanol concentrations below $50 \%{ }^{40}$ This allows an IOF with a particular surface energy to wet at a specific ethanol concentration. To differentiate a series of ethanol concentrations with the same sensor, the IOF should contain several 
spatially separated regions with different surface energies. The maximum resolution using this approach is set by the inherent variability of the neck angles between pores $^{23,24}$ and the number of regions with different surface energies.

One effective method to modify the surface energy of the IOF is functionalization of the surface using silane molecules. Each ethanol threshold (Figure 1e) needs its own unique silane functionalization to distinguish the contact angles $\theta_{c}$ of two ethanol concentrations (e.g., 0 and 5\%). For each functionalization, mixtures above a certain ethanol concentration have contact angles below $\varphi_{0}$ and thus wet the surface, while mixtures below that concentrations have contact angles greater than $\varphi_{0}$, and thus leave the IOF dry and colored. We used 2-[methoxy(polyethyleneoxy)9-12propyl]trimethoxysilane (PEG) and acetoxyethyldimethylchlorosilane (ACE) to obtain regions of different hydrophobicity. We applied the silanes by submerging the substrate in a $1 \%$ solution of the silanes in toluene (see experimental section for details). The contact angle $\theta_{c}$ of pure water on PEG-treated glass was about $23^{\circ}$, slightly larger than the threshold $\varphi_{0}=21^{\circ}$. The contact angle of a $40 \%$ ethanol-water mixture on ACE-treated glass was about $26^{\circ}$, while the contact angle of pure water on ACE-treated glass reaches $67^{\circ}$ (Figure S1). Therefore, the PEG-treated and ACE-treated IOF are expected to stay non-wetted and colorful in pure water and in up to $40 \%$ ethanol solution, respectively.

To distinguish ethanol concentrations between 0 and 40\%, we prepared PEG and ACE mixtures to functionalize glass surfaces, which changed the contact angle of ethanol solutions proportionally to the ratio of ACE to PEG (Figure 2b). As we increased the amount of hydrophilic PEG in the mixture, the contact angle gradually decreased for any given ethanol concentration, with the lowest contact angles corresponding to high ethanol concentrations. We extracted appropriate ratios of ACE to PEG for ethanol concentrations between $0-5 \%, 5-10 \%, 10-20 \%, 20-30 \%, 30-40 \%$ and $>40 \%$ (Table S1) by comparing the contact angle variations to the wetting threshold $\varphi_{0}=21^{\circ}$ (red dashed line, Figure 2b).

In order to make an all-in-one ethanol sensor capable of reporting a broad range of ethanol concentrations, we applied these silane mixtures onto an IOF substrate by sequentially dip-coating the 
IOF in $1 \%$ solutions of the corresponding mixtures of silanes in toluene (Figure 3a). We controlled the size of the coated section by adjusting the immersion height. To prevent cross-contamination, we first analyzed the relative stability of the PEG- and ACE-functionalized surfaces when exposed to the solution of the other silane. Our results show that while ACE reacts with a PEGylated surface, changing its wettability, immersion of the ACE-functionalized substrate into a PEG solution does not affect the surface wettability of the previously functionalized region. This is likely due to the fact that the ACE molecule is mono-chlorinated and therefore does not provide additional crosslinking sites, whereas PEG bears three methoxy groups capable of reacting with incoming ACE molecules. As a result, an ACEcoated IOF showed no change in infiltration after PEG-treatment, while a PEG-coated IOF treated with ACE did not maintain its hydrophilicity (Figure 3b). Therefore, to create a single sensor with well-defined and chemically stable regions of variable hydrophobicity, we started the treatment with the most hydrophobic ACE solution and sequentially dipped the substrate in progressively more hydrophilic PEGcontaining solutions.

Based on the silane ratios discussed previously, we fabricated a colorimetric indicator using a $4.5 \times$ $0.8 \mathrm{~cm}$ IOF coated silicon wafer. The IOF sensor was prepared from six dip-coating solutions to show seven distinct segments that exhibit increasing hydrophobicity (Figure 3c). The first segment wet in pure water, while subsequent segments required increasing concentrations of ethanol, where a 5\% solution would wet the first two segments, $10 \%$ the first three and so on, up to $40 \%$ ethanol where all but the last segment would be wet (Figure 3d). As segments lose their coloration upon wetting, each ethanol concentration is easily identified by the boundary between the segments. These boundaries matched well with the contact angle measurements on glass (Figure $2 b$ ) and comply with our design goals (Figure 1e). In contrast to conventional colorimetric sensors which have slow response and non-intuitive readout, our sensor offers a well-defined readout (colorful or not) within a few seconds. The clean wetting edge further facilitates the accuracy and the ease of reading. Moreover, this indicator can be easily reused by simply drying the IOF, which is another highly preferred feature for on-site operation. The sensing resolution can 
be further improved by tuning the compositions of the silane mixtures and introducing horizontal and vertical chemical gradients as described by Burgess et al $;{ }^{24}$ at this stage, however, our study is aimed at developing a simple, low-cost, easy-to-use sensor, which the approach described here is likely to achieve.

To demonstrate the versatility of the sensor, we seamlessly integrated this technique with typical household glassware, exemplified by a glass stirrer and a glass vial. Through a slightly modified coassembly process (see experimental details), we obtained a uniform and colorful IOF thin film on a glass rod (Figure 4a). The IOF surface was then dip-coated with a selection of our previously used silane mixtures. Thus treated, the stirrer is capable of differentiating water, $10 \%, 20 \%$ and $30 \%$ ethanol with quick response and clear readout (Figure 4b). We also applied the iridescent IOF and the silane chemistry onto a glass vial and demonstrated an iridescent vial that can immediately report the ethanol concentrations (Figure 4c). These results show that our sensor is highly practical to indicate ethanol concentration. Additives in commercial drinks may impact the surface tension. However, the low concentrations typical for common additives (e.g., sugars and other organic molecules) are unlikely to change the surface tension by more than $5 \%,{ }^{41}$ which is within the intrinsic detection limit of this sensor. The additives would preferentially stay in the liquid rather than being adsorbed on the surface of the sensor, as entropy gain due to solvation will likely overcome the weak van der Waals interaction between the additives and the saturated carbon tails of the silanes.

\section{Conclusion}

In summary, we have developed a reusable colorimetric indicator for low-concentration ethanol detection based on the coupling of wetting and structural coloration. The colorful iridescence produced by a highly ordered silica inverse opal film provides an optical signal that is a product of liquid infiltration controlled by the surface functionalization of the IOF. We used a series of silane mixtures of PEG and ACE to discern ethanol concentrations ranging from 0 to $40 \%$. With these mixtures, we fabricated an allin-one ethanol sensor with preassigned segments corresponding to distinct ethanol concentrations, which 
enables quick, straightforward evaluation of ethanol concentrations. This colorimetric sensing scheme was then seamlessly integrated onto traditional glassware, demonstrating the versatility of our approach.

\section{Associated Content}

\section{Supporting Information}

Experimental details, a supporting figure showing the contact angle of water on PEG-treated glass, $40 \%$

EtOH on ACE-treated glass, water on ACE-treated glass, a supporting table showing the silane ratios for different ethanol concentrations.

\section{Author Information}

\section{Corresponding Author}

*E-mail: jaiz@seas.harvard.edu

\section{ORCID}

Yanhao Yu: 0000-0003-2494-3006

Soeren Brandt: 0000-0002-6020-7340

Natalie J. Nicolas: 0000-0003-2489-2448

Joanna Aizenberg: 0000-0002-2343-8705

\section{Notes}

The authors declare no competing financial interest.

\section{Acknowledgement}

This work was supported by the National Science Foundation through the Harvard Materials Research Science and Engineering Center DMR-1420570.

\section{Reference}

1. Rehm, J.; Gmel, G., Sr.; Hasan, O. S. M.; Imtiaz, S.; Popova, S.; Probst, C.; Roerecke, M.; Room, R.; Samokhvalov, A. V.; Shield, K. D.; Shuper, P. A. The Contribution of Unrecorded Alcohol to Health Harm. Addiction 2017, 112, 1687-1688.

2. Thungon, P. D.; Kakoti, A.; Ngashangva, L.; Goswami, P. Advances in Developing Rapid, Reliable and Portable Detection Systems for Alcohol. Biosens. Bioelectron. 2017, 97, 83-99.

3. Hingson, R.; White, A. New Research Findings since the 2007 Surgeon General's Call to Action to Prevent and Reduce Underage Drinking: A Review. J. Stud. Alcohol Drugs 2014, 75, 158-169.

4. Nargiso, J. E.; Friend, K. B.; Egan, C.; Florin, P.; Stevenson, J.; Amodei, B.; Barovier, L. Coalitional Capacities and Environmental Strategies to Prevent Underage Drinking. Am. J. Community Psychol. 2013, 51, 222-231. 
5. Weatherly, C. A.; Woods, R. M.; Armstrong, D. W. Rapid Analysis of Ethanol and Water in Commercial Products Using Ionic Liquid Capillary Gas Chromatography with Thermal Conductivity Detection and/or Barrier Discharge Ionization Detection. J. Agric. Food Chem. 2014, 62, 1832-1838.

6. Talebi, M.; Frink, L. A.; Patil, R. A.; Armstrong, D. W. Examination of The Varied and Changing Ethanol Content of Commercial Kombucha Products. Food Anal. Methods 2017, 10, 4062-4067.

7. Yarita, T.; Nakajima, R.; Otsuka, S.; Ihara, T.; Takatsu, A.; Shibukawa, M. Determination of Ethanol in Alcoholic Beverages by High-Performance Liquid Chromatography-Flame Ionization Detection using Pure Water as Mobile Phase. J. Chromatogr. A 2002, 976, 387-391.

8. Ledauphin, J.; Le Milbeau, C.; Barillier, D.; Hennequin, D. Differences in the Volatile Compositions of French Labeled Brandies (Armagnac, Calvados, Cognac, and Mirabelle) using GC-MS and PLSDA. J. Agric. Food Chem. 2010, 58, 7782-7793.

9. Stupak, M.; Kocourek, V.; Kolouchova, I.; Hajslova, J. Rapid Approach for the Determination of Alcoholic Strength and Overall Quality Check of Various Spirit Drinks and Wines using GC-MS. Food Control 2017, 80, 307-313.

10. Mignani, A. G.; Ciaccheri, L.; Gordillo, B.; Mencaglia, A. A.; González-Miret, M. L.; Heredia, F. J.; Culshaw, B. Identifying the Production Region of Single-Malt Scotch Whiskies using Optical Spectroscopy and Pattern Recognition Techniques. Sens. Actuators, B 2012, 171, 458-462.

11. Martins, A. R.; Talhavini, M.; Vieira, M. L.; Zacca, J. J.; Braga, J. W. Discrimination of Whisky Brands and Counterfeit Identification by UV-Vis Spectroscopy and Multivariate Data Analysis. Food Chem. 2017, 229, 142-151.

12. Smyth, H.; Cozzolino, D. Instrumental Methods (Spectroscopy, Electronic Nose, and Tongue) as Tools to Predict Taste and Aroma in Beverages: Advantages and Limitations. Chem. Rev. 2013, 113, 1429-1440.

13. Askim, J. R.; Mahmoudi, M.; Suslick, K. S. Optical Sensor Arrays for Chemical Sensing: the Optoelectronic Nose. Chem. Soc. Rev. 2013, 42, 8649-8682.

14. Li, Z.; Suslick, K. S. A Hand-Held Optoelectronic Nose for the Identification of Liquors ACS Sens. 2018, 3, 121-127.

15. Li, Z.; Chang, X.; Wang, Y.; Wei, C.; Wang, J.; Ai, K.; Zhang, Y.; Lu, L. Point-and-Shoot Strategy for Identification of Alcoholic Beverages Anal. Chem. 2018, 90, 9838-9844.

16. Li, Z.; Fang, M.; LaGasse, M. K.; Askim, J. R.; Suslick, K. S. Colorimetric Recognition of Aldehydes and Ketones Angew Chem. Int. Ed. Engl. 2017, 56, 9860-9863.

17. Zhang, L.; Qi, H.; Wang, Y.; Yang, L.; Yu, P.; Mao, L. Effective Visualization Assay for Alcohol Content Sensing and Methanol Differentiation with Solvent Stimuli-Responsive Supramolecular Ionic Materials. Anal. Chem. 2014, 86, 7280-7285.

18. Deng, J.; Shi, G.; Zhou, T. Colorimetric Assay for on-the-Spot Alcoholic Strength Sensing in Spirit Samples Based on Dual-Responsive Lanthanide Coordination Polymer Particles with Ratiometric Fluorescence. Anal. Chim. Acta. 2016, 942, 96-103.

19. Deng, J.; Ma, W.; Yu, P.; Mao, L. Colorimetric and Fluorescent Dual Mode Sensing of Alcoholic Strength in Spirit Samples with Stimuli-Responsive Infinite Coordination Polymers. Anal. Chem. 2015, 87, 6958-6965.

20. Kinoshita, S.; Yoshioka, S.; Miyazaki, J. Physics of Structural Colors. Rep. Prog. Phys. 2008, 71, 076401.

21. Fenzl, C.; Hirsch, T.; Wolfbeis, O. S. Photonic Crystal Based Sensor for Organic Solvents and for Solvent-Water Mixtures. Sensors 2012, 12, 16954-16963.

22. Choi, S. Y.; Mamak, M.; von Freymann, G.; Chopra, N.; Ozin, G. A. Mesoporous Bragg Stack Color Tunable Sensors. Nano Lett. 2006, 6, 2456-2461.

23. Burgess, I. B.; Mishchenko, L.; Hatton, B. D.; Kolle, M.; Loncar, M.; Aizenberg, J. Encoding Complex Wettability Patterns in Chemically Functionalized 3D Photonic Crystals. J. Am. Chem. Soc. 2011, 133, 12430-12432.

24. Burgess, I. B.; Koay, N.; Raymond, K. P.; Kolle, M.; Loncar, M.; Aizenberg, J. Wetting in Color: Colorimetric Differentiation of Organic Liquids with High Selectivity. ACS Nano 2012, 6, 1427-1437. 
25. Burgess, I. B.; Lončar, M.; Aizenberg, J. Structural Colour in Colourimetric Sensors and Indicators. $J$. Mater. Chem. C 2013, 1, 6075-6086.

26. Raymond, K. P.; Burgess, I. B.; Kinney, M. H.; Loncar, M.; Aizenberg, J. Combinatorial Wetting in Colour: an Optofluidic Nose. Lab Chip 2012, 12, 3666-3669.

27. Hong, W.; Li, H.; Hu, X.; Zhao, B.; Zhang, F.; Zhang, D. Independent Multifunctional Detection by Wettability Controlled Inverse Opal Hydrogels. Chem. Commun. 2012, 48, 4609-4611.

28. Singleton, T. A.; Burgess, I. B.; Nerger, B. A.; Goulet-Hanssens, A.; Koay, N.; Barrett, C. J.; Aizenberg, J. Photo-Tuning of Highly Selective Wetting in Inverse Opals. Soft Matter 2014, 10, $1325-1328$.

29. Wang, Z.; Liu, X.; Wu, Y.; Liu, B.; Wang, Z.; Zhang, J.; Liu, K.; Yang, B. Ultrathin StimuliResponsive Polymer Film-Based Optical Sensor for Fast and Visual Detection of Hazardous Organic Solvents. J. Mater. Chem. C 2018, 6, 10861-10869.

30. Wu, P.; Guo, J.; Jiang, K.; Wang, J.; Jiang, L. Direct Water-Writing/Electroerasing Pattern on PEDOT Inverse Opals Adv. Funct. Mater. 2019, 29, 1808473.

31. Zhong, K.; Li, J.; Liu, L.; Van Cleuvenbergen, S.; Song, K.; Clays, K. Instantaneous, Simple, and Reversible Revealing of Invisible Patterns Encrypted in Robust Hollow Sphere Colloidal Photonic Crystals Adv. Mater. 2018, 30, e1707246.

32. Xu, Q.; Mahpeykar, S. M.; Burgess, I. B.; Wang, X. Inverse Opal Photonic Crystals as an Optofluidic Platform for Fast Analysis of Hydrocarbon Mixtures ACS Appl. Mater. Interfaces 2018, 10, 2012020127.

33. Li, Y.; Zhou, X.; Yang, Q.; Li, Y.; Li, W.; Li, H.; Chen, S.; Li, M.; Song, Y. Patterned Photonic Crystals for Hiding Information. J. Mater. Chem. C 2017, 5, 4621-4628.

34. Hong, W.; Li, H.; Hu, X.; Zhao, B.; Zhang, F.; Zhang, D.; Xu, Z. Wettability Gradient Colorimetric Sensing by Amphiphilic Molecular Response. Chem. Commun. 2013, 49, 728-730.

35. Aguirre, C.; Reguera, E.; Stein, A. Tunable Colors in Opals and Inverse Opal Photonic Crystals. $A d v$. Funct. Mater. 2010, 20, 2565-2578.

36. Ozin, G. A.; Arsenault A. C. P-Ink and Elast-Ink from lab to market. Mater. Today 2008, 11, 44-51.

37. Phillips, K. R.; England, G. T.; Sunny S.; Shirman, E.; Shirman, T.; Vogel, N.; Aizenberg, J. A colloidoscope of colloid-based porous materials and their uses. Chem. Soc. Rev. 2016, 45, 281-322.

38. Hatton, B.; Mishchenko, L.; Davis, S.; Sandhage, K. H.; Aizenberg, J. Assembly of Large-Area, Highly Ordered, Crack-Free Inverse Opal Films. Proc. Natl. Acad. Sci. USA 2010, 107, 10354-10359.

39. Vogel, N.; Utech, S.; England, G. T.; Shirman, T.; Phillips K. R.; Koay, N.; Burgess, I. B.; Kolle, M.; Weitz, D. A.; Aizenberg, J. (2015). Color from hierarchy: Diverse optical properties of micron-sized spherical colloidal assemblies. Proc. Natl. Acad. Sci. USA 2015, 112,10845-10850.

40. Vazquez, G.; Alvarez, E.; Navaza, J. M. Surface Tension of Alcohol Water + Water from 20 to 50 .degree.C. J. Chem. Eng. Data 1995, 40, 611-614.

41. Serghat, S; Mathlouthi, M.; Hoopman, T.; Birch, G. G. Solute-solvent interactions and the sweet taste of small carbohydrates. Part 1: Effect of solvent polarity on solution properties. Food Chem. 1992, 45, 25-32. 
Figures
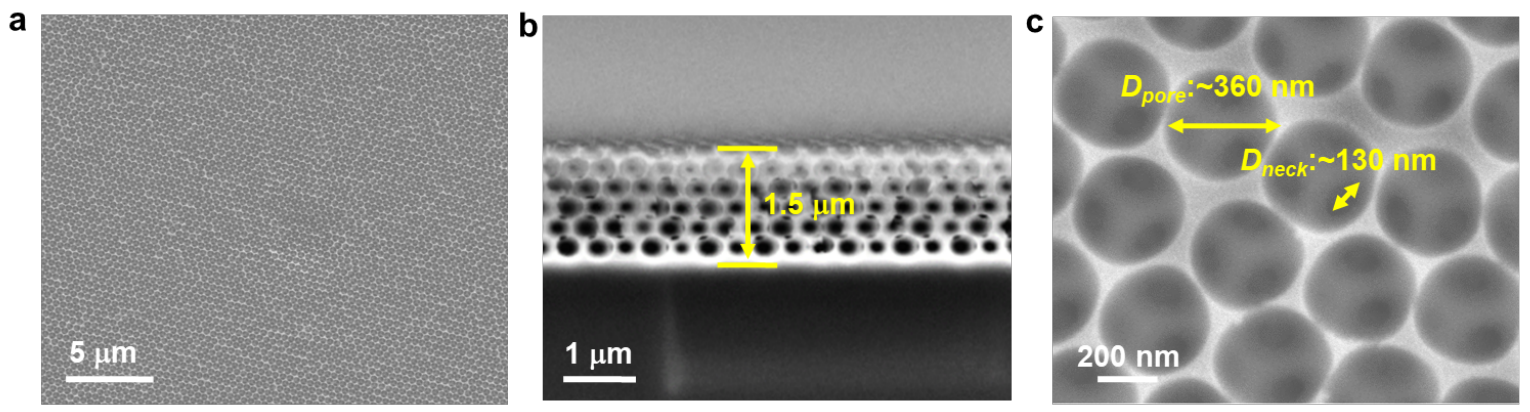

d

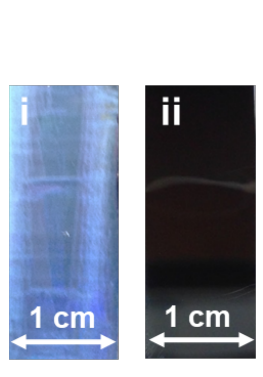

In air

In water

e
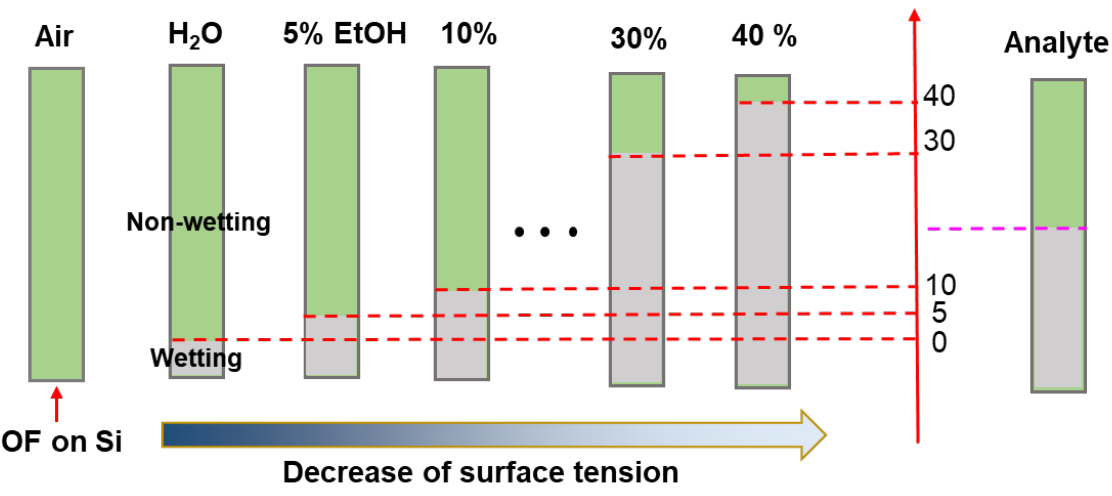

Figure 1. Characterizations of the inverse opal film (IOF) and the design of the ethanol indicator. (a) A top view SEM of the IOF showing the highly ordered and crack-free features of the IOF. (b) A crosssectional view SEM of the IOF that identifies the five and a half inverse opal layers yielding a film thickness of $1.5 \mu \mathrm{m}$. (c) A high-magnification top view SEM of the IOF that illustrates the sizes of pores and necks. (d) Two digital images manifest the strong iridescence of IOF in air (i) and the disappearance of color in water (ii). (e) The operational principle of the colorimetric ethanol indicator based on the coupling of wetting and structural coloration 
a

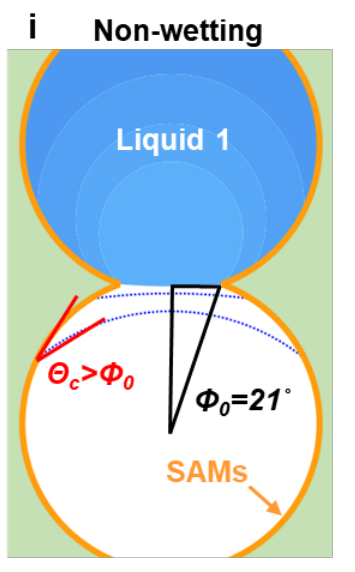

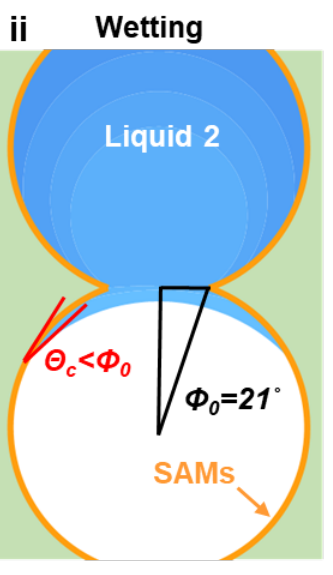

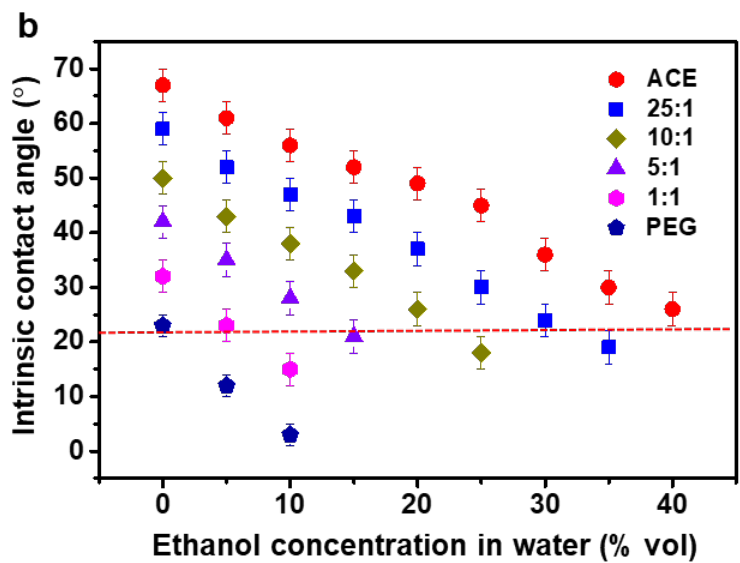

Figure 2. Development of the silane chemistry for IOF functionalization. (a) Schematic illustrations of the relationship between the contact angle $\left(\theta_{c}\right)$ and the neck opening angle $\left(\varphi_{0}\right)$ when IOF is non-wetted (i) and wetted (ii). (b) Measured contact angles as a function of the ethanol concentrations in water on flat glass surfaces after treated by PEG, ACE and their mixtures with varied ratios. The ratios are all volumetric ACE to PEG ratios. For instance, 25:1 means that the ratio of ACE to PEG volumes used equals to $25: 1$. The red dashed line marks the wetting threshold determined by $\varphi_{0}$. 
a

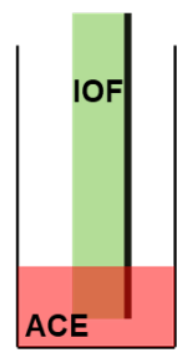

Step I

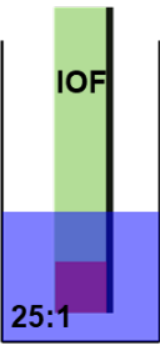

Step II b

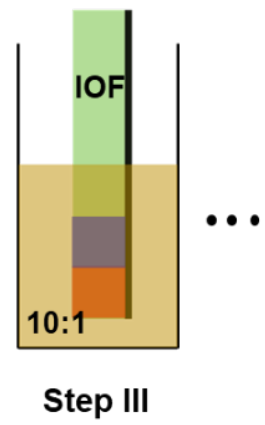

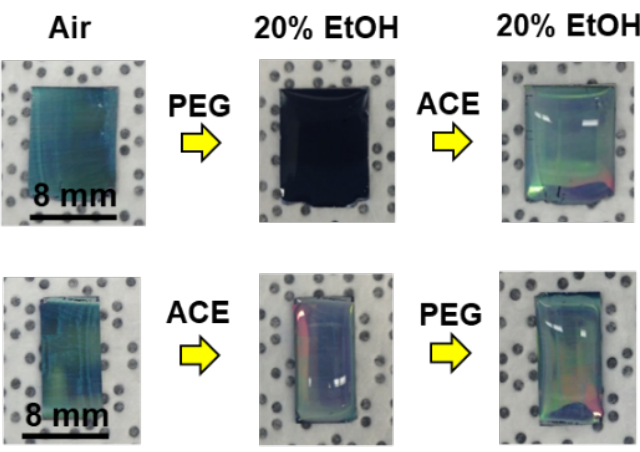

EtOH concentration (\%)

C

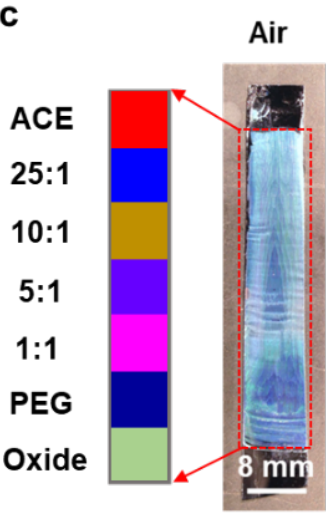

d

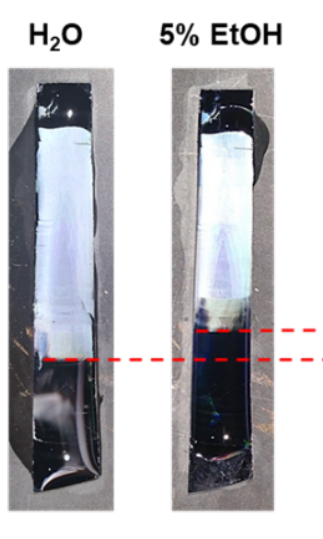

$30 \% \quad 40 \%$ EtOH

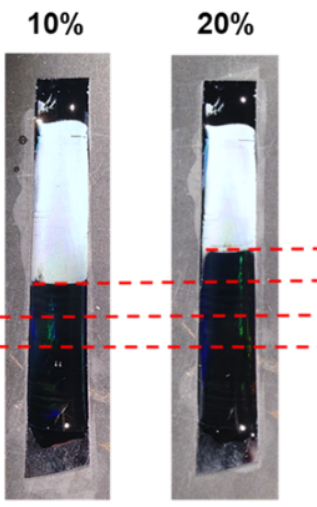

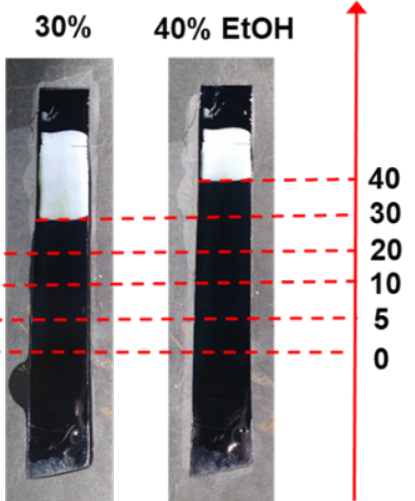

Figure 3. Dip-coating silanes onto IOF and demonstration of the function of the ethanol indicator. (a) Schematic illustrations of the dip-coating process that sequentially deposits silanes onto different sections of IOF. (b) Digital images showing that PEG coating is unstable upon ACE interference while ACE can endure the PEG treatment. (c) A schematic and corresponding digital image showing the surface chemistries of each segments on the IOF. (d) The wetting and color behaviors of the ethanol indicator (the one presented in Figure 3c) in different ethanol concentrations, demonstrating the ethanol detecting capability with clear readout. 


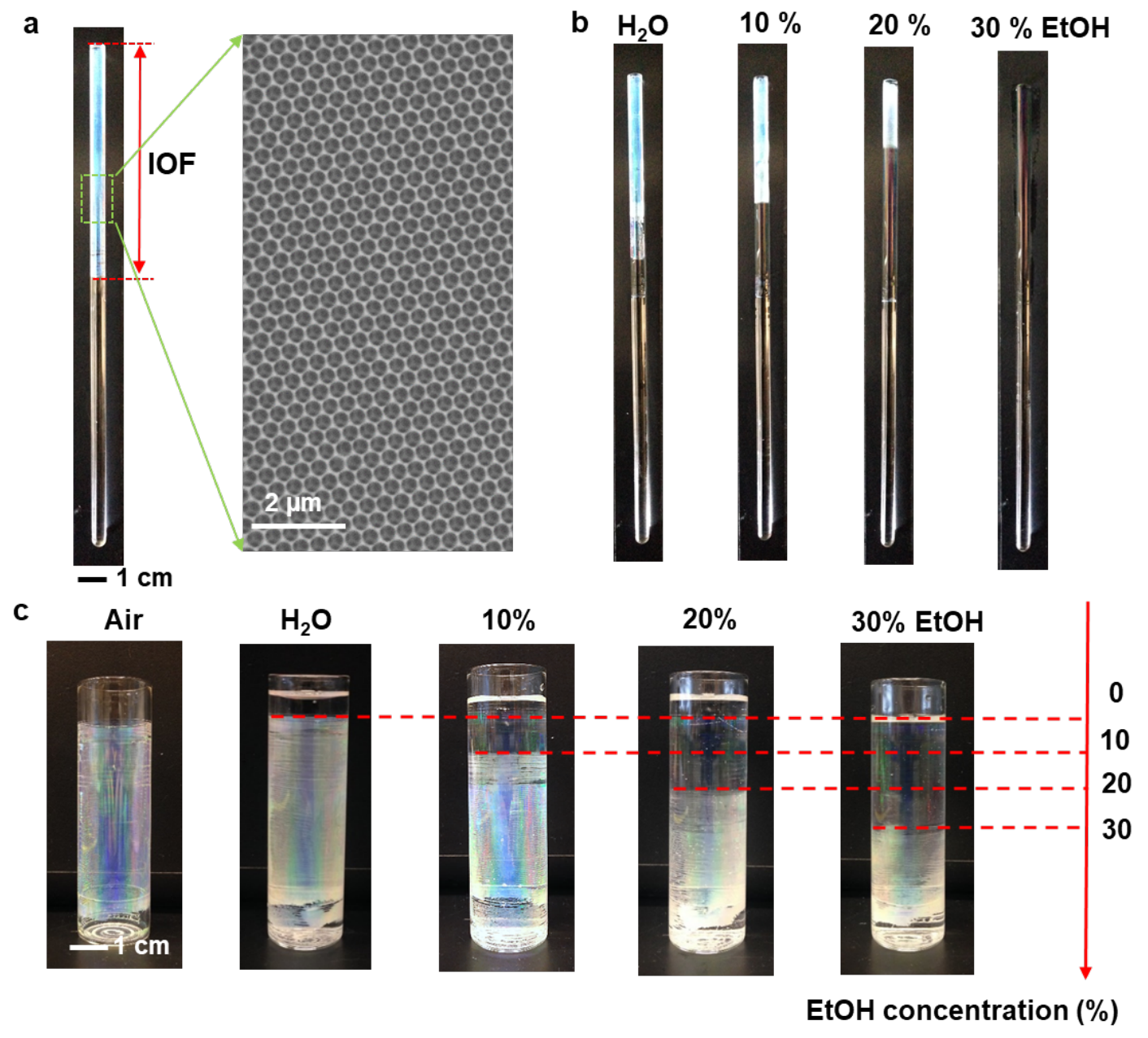

Figure 4. Field-ready ethanol indicators integrated on a glass stirrer and a glass vial. (a) A digital image of the glass stirrer whose top half is coated with silane-treated IOF. The SEM image shows the high uniformity of the IOF. (b) Digital images illustrate the gradual increase of the wetting segments and related decrease of the iridescent segments with the increment of ethanol concentrations, which serve as an easy-to-read optical signal for ethanol detection. (c) An iridescent glass vial that can discern ethanol concentrations based on a concept similar to the one demonstrated in Figure 3d and Figure 4b. 
Table of Contents

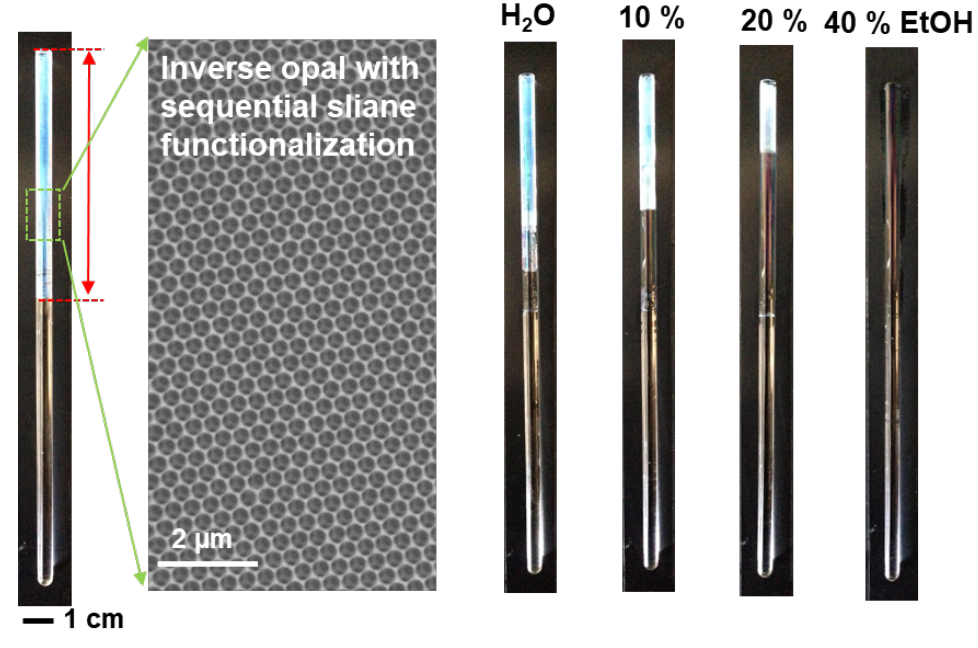

